\title{
ORIGINAL ARTICLE \\ Intradetrusor versus suburothelial onabotulinumtoxinA injections for neurogenic detrusor overactivity: a pilot study
}

\author{
J Krhut $^{1}$, V Samal ${ }^{2}$, D Nemec ${ }^{1}$ and P Zvara ${ }^{3}$
}

Study design: This was a multicentre, prospective, randomised study.

Objectives: To compare the outcomes of intradetrusor and suburothelial onabotulinumtoxinA injections in patients with spinal cord injury and refractory neurogenic detrusor overactivity (NDO).

Setting: Urology departments of two tertiary hospitals in the Czech Republic.

Methods: A total of 32 spinal cord injury patients with severe NDO refractory to the standard anticholinergic treatment were randomised to receive either intradetrusor or suburothelial $300 \mathrm{IU}$ onabotulinumtoxinA injections. Subjective satisfaction, bladder diary data and urodynamic data were compared in both groups before treatment and at 3 months post treatment.

Results: In all, $64.3 \%$ patients in the intradetrusor group and $88.8 \%$ patients in the suburothelial group were subjectively satisfied with the treatment. There was a significant post-treatment improvement in both groups regarding the number of catheterisations over $24 \mathrm{~h}$, number of incontinence episodes over $24 \mathrm{~h}$, catheterised volume, cystometric capacity, volume at first involuntary detrusor contraction, maximal detrusor pressure during filling and detrusor compliance. No significant differences between the groups were observed, with the exception of improvement of detrusor compliance, which was better in the intradetrusor group. There was one adverse effect comprising transient muscle weakness that was reported by one patient in the intradetrusor group.

Conclusion: Results in both groups were comparable. The authors favour suburothelial onabotulinumtoxinA injection because this method allows more precise toxin localisation.

Spinal Cord (2012) 50, 904-907; doi:10.1038/sc.2012.76; published online 17 July 2012

Keywords: urodynamics; neurogenic detrusor overactivity; onabotulinumtoxinA; spinal cord injury; suburothelial injection

\section{INTRODUCTION}

Spinal cord injuries are among the most devastating of all injuries, often having life-changing and diverse consequences. Lower urinary tract dysfunction is commonly observed as a result of a spinal cord injury. Neurogenic detrusor overactivity (NDO) is frequently associated with upper motor neuron lesion, and the subsequent increased intravesical pressure may present a potential risk by causing damage to the upper urinary tract. ${ }^{1}$ Current standard NDO treatment comprises individualised administration of anticholinergic medication; however, this therapy is inadequate for some patients and is often associated with unacceptable adverse effects. ${ }^{2}$ Treatment with botulinum toxin offers an accepted alternative treatment for those patients in whom anticholinergic treatment has failed.

Botulinum toxin is a neurotoxin produced by anaerobic microorganisms of the Clostridium genus. Botulinum toxin acts on the peripheral nervous system where it is responsible for blockade of acetylcholine release from presynaptic nerve endings. ${ }^{3}$ This process results in blockade of the neuromuscular transfer, with a subsequent loss of muscle cell contractility.

A further effect of botulinum toxin following administration into the urinary bladder wall is an inhibitory influence on neurotransmitters and receptors mediating sensory neurotransmission., ${ }^{4,5}$

The clinical effect of botulinum toxin injection in the treatment of NDO has been established in several studies. ${ }^{6-8}$ Further aspects of this form of treatment are currently under discussion, such as optimal dose, optimal dilution of botulinum toxin and the most effective administration technique (for example, bladder trigonum-saving technique versus injection into bladder trigonum).

The lamina propria sensory nerves probably have a key role in the pathophysiology of the detrusor overactivity. ${ }^{9}$ Therefore, the suburothelial injection of the onabotulinumA seems to be the most logical application area. However, most treatment protocols published untill now favour intradetrusor application. The aim of this multicentre, prospective randomised trial was to compare the efficacy and safety of intradetrusor and suburothelial injection of onabotulinumtoxinA in a group of patients with NDO caused by a spinal lesion.

\section{MATERIALS AND METHODS}

This was a multicentre, randomised, prospective study comprising two groups of patients. Patients in group A received onabotulinumtoxinA injections into the detrusor, whereas patients in group B received onabotulinumtoxinA suburothelial injections. This study was approved by the local Ethics Committee, and all the enrolled patients signed informed consent before any study-specific procedure. All applicable institutional and governmental regulations concerning the ethical use of human volunteers were followed during the course of this research.

A total of 32 patients were enrolled into the study (26 males and 6 females), with a mean age of 32.1 years (range: $20-55$ years). The main demographic

${ }^{1}$ Department of Urology, University Hospital, Ostrava, Czech Republic; ${ }^{2}$ Department of Urology, Regional Hospital, Liberec, Czech Republic and ${ }^{3}$ Division of Urology, University of Vermont, Burlington, VT, USA

Correspondence: Professor J Krhut, Department of Urology, University Hospital Ostrava, 17 Listopadu 1790, 70852 Ostrava, Czech Republic.

E-mail: jan.krhut@fnspo.cz

Received 21 November 2011; revised 26 April 2012; accepted 27 April 2012; published online 17 July 2012 
characteristics of the study population are provided in Table 1. Patient inclusion criteria comprised NDO as evidenced urodynamically. Patients were required to be neurologically stable, with failure of oral anticholinergic treatment due to insufficient efficacy or adverse effects, and the need for urinary bladder evacuation with intermittent catheterisation (that is, autocatheterisation or catheterisation by another person).

Patients with a history of botulinum toxin treatment, patients with cystolithiasis or previous surgery, or a history of radiation treatment in the pelvic region, together with females of child-bearing potential who were not using reliable methods of contraception, as well as breastfeeding females, were all excluded from the study.

The following parameters were monitored during the course of the study: subjective satisfaction, parameters obtained following analysis of the bladder diary and the results of urodynamic examination. All the parameters were evaluated immediately before treatment and at 3 months post treatment. Patients were then followed-up until symptoms returned to the baseline level.

\section{Urodynamic measurement}

Urodynamic evaluation was performed according to the International Continence Society recommendations, with the following additions: (a) volume at first involuntary detrusor contraction ( $\mathrm{Vol}$ at $\mathrm{FC}$ ) and (b) maximal detrusor pressure during filling $\left(\mathrm{p}_{\operatorname{det}} \mathrm{F}\right)$ representing the highest amplitude of nonvoiding contraction. ${ }^{10}$ Values were recorded from patients in the supine position using a filling rate of $50 \mathrm{ml} \mathrm{min}^{-1}$.

\section{Injection procedure}

Patients were treated with onabotulinumtoxinA (Botox; Allergan Inc., Irvine, CA, USA) at a dosage of $300 \mathrm{IU}$, diluted in $30 \mathrm{ml}$ of saline. Furthermore, all patients received antibiotic treatment prior to the procedure to ensure urine sterility. The procedure was performed under general anaesthesia, with bladder filling at approximately $200 \mathrm{ml}$. Sonographic measurement of the thickness of the bladder wall preceded application of onabotulinumtoxinA. Onabotulinumtoxin A was administered with $25 \mathrm{G}$ endoscopic needle into 30 locations so that the whole endoscopically accessible area of the bladder wall was evenly covered. A dose of $1 \mathrm{ml}$ onabotulinumtoxinA was applied with each puncture.

In group $\mathrm{A}$, onabotulinumtoxin $\mathrm{A}$ was injected at a depth of approximately 2-4 mm, depending on the thickness of the bladder wall as measured by ultrasound. No visual bulking of the mucosa was observed upon injecting, and muscle tissue resistance was felt during administration. In cases when there was only a slight or no backpressure of the syringe plunger, toxin administration

Table 1 Demographic characteristics of the study population

\begin{tabular}{lcc} 
& Group A & Group B \\
\hline Total number & 14 & 18 \\
Males & 9 & 17 \\
Females & 5 & 1 \\
Age (years) & 31.8 (range: 20-48) & 32.4 (range: 20-55) \\
BMI & 25.6 (range: 20-32) & 25.2 (range: 18-31) \\
& & \\
Lesion localisation & & 8 \\
C & 8 & 9 \\
Th & 5 & 1 \\
L & 1 & \\
ASIA & & 14 \\
A & & 3 \\
B & 10 & 1 \\
C & 3 & 0 \\
D & 1 & 0 \\
E & 0 & \\
& 0 &
\end{tabular}

Time from injury (months) 58.8 (range: 16-154) 60.7 (range: 11-276)

Abbreviations: ASIA: American Spinal Injury Association; BMI: body mass index. was discontinued immediately as we suspected possible localisation of the needle tip into the thin paravesical adipose tissue. In these cases, the needle tip was removed from the bladder wall and inserted into a different part of the bladder wall.

Patients in group B received onabotulinumtoxinA injected into the submucosa in such a way that the bulking was clearly visible. A bladder drainage with permanent catheter was maintained for a period of $24 \mathrm{~h}$ following toxin administration, after that time the patients continued with evacuation via intermittent catheterisation.

\section{Statistical analysis}

The data are expressed as means \pm s.e.m. Statistical analyses were carried out using paired Student's $t$-tests. In cases where data were not normally distributed, a Wilcoxon signed-rank test was used. ${ }^{11} P$-values $\leqslant 0.05$ were considered statistically significant.

\section{RESULTS}

To assess subjective satisfaction, all patients were asked whether they were satisfied with onabotulinumtoxinA treatment to the extent that they would be willing to undergo repeat treatment once all the beneficial effects of the first treatment had diminished. A total of 9 (64.3\%) patients in group A requested repeat treatment, 2 (14.3\%) patients were undecided and $3(21.4 \%)$ patients stated they would refuse repetition of the treatment procedure. In group B, $16(88.8 \%)$ patients were willing to undergo repeat treatment, 1 (5.6\%) patient was undecided and $1(5.6 \%)$ patient would refuse repetition of the treatment.

Based upon the information from bladder diaries, the frequency of urinary bladder catheterisation, the number of incontinence episodes and catheterised volume for each patient was assessed. All these parameters in both study groups were significantly improved after treatment, and the differences observed between groups A and B were not statistically significant.

On the basis of the data obtained from urodynamic examination, cystometric capacity, volume at first involuntary detrusor contraction, maximal detrusor pressure and detrusor compliance were also evaluated. All the parameters in both groups showed a statistically significant improvement following onabotulinumtoxinA treatment. One parameter showed a statistical difference between the two treatment groups, with detrusor compliance being statistically significantly better in group A. No statistically significant difference between the two groups was noted for any of the other parameters (Table 2).

There was no statistically significant difference between the two treatment groups with regard to duration of effect of treatment. Treatment duration was 7.29 \pm 1.27 (range 5-9) months in group A and $7.11 \pm 1.31$ (range $5-9)$ months in group B $(P=0.726)$.

There was only one treatment-related adverse effect observed in one patient from group A during the study period. The patient complained of a loss of muscle strength for a period of 7 days following onabotulinumtoxinA administration. This adverse effect resolved completely and the patient made a full recovery.

\section{DISCUSSION}

OnabotulinumtoxinA injection into the urinary bladder wall presents an effective alternative treatment for NDO refractory to the standard treatment with anticholinergic medication. The effectiveness and safety of this treatment has been assessed in a number of clinical trials. $^{12}$

The clinical effect of botulinum toxin results from a temporary chemical denervation in the target application area. ${ }^{13}$ Axonal resprouting follows after several weeks or months, depending upon 
the dose of botulinum toxin administered. However, according to a study by Haferkamp et al., ${ }^{14}$ only a limited resprouting is seen in detrusor biopsies compared with striated muscle. Axonal resprouting was noted in only three axons of 11 detrusor biopsies in patients already experiencing diminished clinical effects of botulinum toxin injections. Thus, the data show that the duration of clinical effect does not depend on the timing of detrusor reinnervation.

Alternative data suggest that the local action of intradetrusor botulinum toxin injection may effect a functional motor inhibition not associated with neuronal death. Furthermore, botulinum toxin has recently been demonstrated to have inhibitory effects on additional neuronal populations (for example, sensory and autonomic) and non-neuronal tissue (for example, urothelium). ${ }^{15}$ A number of experimental studies in rodents have provided evidence that the sensory effects of botulinum toxin might be a consequence of a suppression of afferent signalling. When injected into the bladder wall, onabotulinumtoxinA demonstrated inhibition of sensory transmitter adenosine triphosphate release, which is released following stimulation of bladder mechanoreceptors. ${ }^{16}$ In addition, afferent nerve-mediated contractions in response to electrical and chemical stimulation were shown to be suppressed in the rat bladder in vitro following botulinum toxin treatment. ${ }^{17}$ The sensory nerves expressing TRPV1, P2X3, substance $\mathrm{P}$ and calcitonin gene-related peptide are most dense in the suburothelial plexus and urothelium. ${ }^{18}$

It seems to us that suburothelial injection is the most logical area for onabotulinumtoxinA application. However, most treatment protocols favour administration of onabotulinumtoxinA into the detrusor. The only experience with suburothelial injection of onabotulinumtoxinA was published by Kuo. ${ }^{19}$ The author reports treatment doses of 100,150 and $200 \mathrm{IU}$ of onabotulinumtoxinA administered suburothelially as having identical effects in patients with refractory idiopathic detrusor overactivity. In another study, $\mathrm{KuO}^{20}$ compared the effect of a $100 \mathrm{IU}$ dose of onabotulinumtoxinA injected into the detrusor, into the base of the bladder and suburothelially in 45 patients with idiopathic detrusor overactivity. A successful result was obtained in 14 (93\%) patients with detrusor, $12(80 \%)$ with suburothelial and $10(67 \%)$ with bladder base injection at 3 months. The success rate decreased over time to $10(67 \%), 7$ $(47 \%)$ and $2(13 \%)$ at 6 months and $3(20 \%), 3(20 \%)$ and $1(6.7 \%)$ at 9 months in the detrusor, suburothelial and bladder base groups, respectively $(P=0.0253){ }^{20}$

To our knowledge, this study is the first to compare the outcomes of intradetrusor and suburothelial onabotulinumtoxinA administration in patients with NDO. Our results show that both the methods of onabotulinumtoxinA injection have the same effect and duration of clinically beneficial effects.

The subjective satisfaction with the treatment was high in both groups. The use of standardised quality-of-life and treatment impact questionaires or visual analagoue scales was not used in this pilot trial. Although superior in reporting the subjective outcomes, the willingness to undergo a retreatment in the case of this study provides sufficient evidence that most patients in both groups considered the treatment a success. The variance between both the groups is more likely due to small number of enrolled patients and is not of statistical significance.

We observed only one treatment-related adverse effect in one patient assigned to group A, comprising a temporary loss of muscle strength. In our opinion, this event may have been caused by unintentional administration of onabotulinumtoxinA into the perivesical space. This hypothesis is supported by Mehnert et al., ${ }^{21}$ in which the authors examined the distribution of botulinum toxin 
labelled with gedopentate using magnetic resonance performed immediately after endoscopic injection. They found $82.4 \%$ of the botulinum toxin located in the detrusor, whereas $17.6 \%$ was situated in the perivesical fat tissue. Similarly, Boy et al. ${ }^{22}$ found that up to $33 \%$ of injected botulinum toxin was not localised in the detrusor.

\section{CONCLUSION}

In conclusion, the therapeutic effects and duration of intradetrusor and suburothelial injection of onabotulinumtoxinA in patients with NDO are comparable.

The authors consider that the suburothelial injection technique is at least as safe as intradetrusor injection technique because it enables precise visual monitoring of the area requiring toxin administration.

\section{DATA ARCHIVING}

There were no data to deposit.

\section{CONFLICT OF INTEREST}

The authors declare no conflict of interest.

\section{ACKNOWLEDGEMENTS}

Medical writing support for this paper was provided by Debbie Jordan, with funding from Allergan Ltd. Statistical analysis was provided by Josef Tvrdik, Ostrava University.

1 Gerridzen RG, Thijssen AM, Dehoux E. Risk factors for upper tract deterioration in chronic spinal cord injury patients. J Urol 1992; 147: 416-418.

2 Wyndaele JJ, Kovindha A, Madersbacher H, Radziszewski P, Ruffion A, Schurch B et al. Committee 10 on Neurogenic Bladder and Bowel of the International Consultation on Incontinence 2008-2009. Neurologic urinary incontinence. Neurourol Urodyn 2010; 29: 159-164.

3 Montecucco C, Schiavo G. Structure and function of tetanus and botulinum neurotoxins. Q Rev Biophys 1995; 28: 423-472.

4 de Paiva A, Meunier FA, Molgo J, Aoki KR, Dolly JO. Functional repair of motor endplates after botulinum neurotoxin type A poisoning: biphasic switch of synaptic activity between nerve sprouts and their parent terminals. Proc Natl Acad Sci USA 1999; 96: 3200-3205.

5 Apostolidis A, Popat R, Yiangou Y, Cockayne D, Ford AP, Davis JB et al. Decreased sensory receptors $\mathrm{P} 2 \mathrm{X} 3$ and TRPV1 in suburothelial nerve fibres following intradetrusor injections of botulinum toxin for human detrusor overactivity. J Urol 2005; 174: 977-982.

6 Reitz A Stöhrer M, Kramer G, Del Popolo G, Chartier-Kastler E, Pannek J et al. European experience of 200 cases treated with botulinum-A toxin injections into the detrusor muscle for urinary incontinence due to neurogenic detrusor overactivity. Eur Urol 2004; 45: 510-515.

7 Bagi P, Biering-Sørensen F. Botulinum toxin A for treatment of neurogenic detrusor overactivity and incontinence in patients with spinal cord lesions. Scand J Urol Nephrol 2004; 38: 495-498.

8 Giannantoni A, Mearini E, Del Zingaro M, Porena M. Six-year follow-up of botulinum toxin $A$ intradetrusorial injections in patients with refractory neurogenic detrusor overactivity: clinical and urodynamic results. Eur Urol 2009; 55: 705-711.

9 Dixon JS, Gilpin CJ. Presumptive sensory axons of the human urinary bladder. A fine ultrastructural study. J Anat 1987; 151: 199-207.

10 Abrams P, Cardozo L, Fall M, Griffiths D, Rosier P, Ulmsten U et al. Standardisation Sub-committee of the International Continence Society. The standardisation of terminology in lower urinary tract function: report from the standardisation sub-committee of the International Continence Society. Urol 2003; 61: 37-49.

11 Hintze J. NCSS and PASS, Number Cruncher Statistical Systems, Kaysville, Utah, USA 2001. www.ncss.com.

12 Leippold T, Reitz A, Schurch B. Botulinum toxin as a new therapy option for voiding disorders: current state of the art. Eur Urol 2003; 44: 165-174.

13 Schiavo G, Matteoli M, Montecucco C. Neurotoxins affecting neuroexocytosis. Physiol Rev 2000; 80: 717-766.

14 Haferkamp A, Schurch B, Reitz A, Krengel U, Grosse J, Krammer G et al. Lack of ultrastructural detrusor changes following endoscopic injection of botulinumtoxin type A in overactive neurogenic bladder. Eur Urol 2004; 46: 784-791.

15 Rapp DE, Lucioni A, Bales GT. Botulinum toxin injection: a review of injection principles and protocols. Int Braz J Urol 2007; 33: 132-141.

16 Smith CP, Gangitano DA, Munoz A, Salas NA, Boone TB, Aoki KR et al. Botulinum toxin type $A$ normalizes alterations in urothelial ATP and NO release induces by chronic spinal cord injury. Neurochem Int 2008; 52: 1068-1075.

17 Chancellor NB, Fowler CJ, Apostolidis A, de Groat WC, Smith CP, Somogyi GT et al. Drug insight: biological effects of botulinum toxin A in the lower urinary tract. Nat Clin Pract Urol 2008; 5: 319-328.

18 Kanai A, Andersson KE. Bladder afferent signaling: recent findings. J Urol 2010; 183 : 1288-1295.

19 Kuo HC. Will suburothelial injection of small dose of botulinum A toxin have similar therapeutic effects and less adverse events for refractory detrusor overactivity? Urol 2005; 66: 94-98.

20 Kuo HC. Comparison of effectiveness of detrusor, suburothelial and bladder base injections of botulinum toxin a for idiopathic detrusor overactivity. J Urol 2007; 178: 1359-1363.

21 Mehnert U, Boy S, Schmid M, Reitz A, von Hessling A, Hodler J et al. A morphological evaluation of botulinum neurotoxin $A$ injections into detrusor muscle using magnetic resonance imaging. World J Urol 2009; 27: 397-409.

22 Boy S, Schmid M, Reitz A, Von Hessling A, Hodler J, Schurch B. Botulinum toxin injections into the bladder wall - a morphological evaluation of the injections technice using magnetic resonance paging. J Urol. Suppl 2006; 175: 415: abstract 1287. 